\title{
Les Peuls, les Sénoufo et l'État au nord de la Côte d'Ivoire. Problèmes fonciers et gestion $\mathrm{du}$ pastoralisme.
}

Youssouf Diallo

\section{(2) OpenEdition \\ Journals}

Édition électronique

URL : http://journals.openedition.org/apad/1131

DOI : 10.4000/apad. 1131

ISSN : 1950-6929

Éditeur

LIT Verlag

Édition imprimée

Date de publication : 1 décembre 1995

Référence électronique

Youssouf Diallo, «Les Peuls, les Sénoufo et l'État au nord de la Côte d'Ivoire. Problèmes fonciers et gestion du pastoralisme. », Bulletin de I'APAD [En ligne], 10 | 1995, mis en ligne le 17 juillet 2007,

consulté le 10 décembre 2020. URL : http://journals.openedition.org/apad/1131 ; DOl : https://doi.org/ 10.4000/apad.1131

Ce document a été généré automatiquement le 10 décembre 2020.

Bulletin de I'APAD 


\title{
Les Peuls, les Sénoufo et l'État au nord de la Côte d'Ivoire. Problèmes fonciers et gestion du pastoralisme.
}

\author{
Youssouf Diallo
}

1 L'expansion pastorale dans le nord de la Côte d'Ivoire est un phénomène relativement récent. Les migrations des Peuls maliens et burkinabé et la demande de viande sans cesse croissante qui ont éveillé l'attention des autorités ivoiriennes vont les pousser ensuite à s'intéresser au secteur de l'élevage. Très vite, l'État décide en effet de recourir aux Peuls pour impulser la production pastorale en mettant en place une politique d'accueil attractive de ces pasteurs désormais encouragés à se fixer en Côte d'Ivoire. Mais l'élevage extensif se heurte aujourd'hui à l'hostilité des paysans sénoufo détenteurs des droits coutumiers des zones d'accueil des éleveurs peuls.

Cette réflexion sur les relations entre les Sénoufo, les Peuls et l'Etat ivoirien concerne principalement le cas du département de Boundiali ${ }^{1}$. Après avoir rappelé les efforts fournis par la Côte d'Ivoire dans le domaine de la production animale, nous nous attacherons à dégager les modalités majeures des relations entre les Peuls et les Sénoufo se trouvant dans une situation de concurrence pour les ressources.

L'économie politique de l'élevage

3 La Côte-d'Ivoire, comme tous les États côtiers ouest-africains (Ghana, Togo, Bénin, Nigeria, "etc.) dépend habituellement de la région sahélo-soudanienne en matière d'approvisionnement en viande. Mais ce pays, qui reste le plus grand importateur de bétail en provenance des pays sahéliens surtout du Burkina Faso et du Mali, présente également la particularité d'être le principal débouché ouest-africain de viande congelée européenne. Pour réduire cette dépendance vis-à-vis du marché extérieur, tant extra-africain (C.E.E., argentine, etc.) qu'africain, les autorités ivoiriennes définissent au début des années 1970 une politique pastorale dans le cadre de laquelle est encouragée la sédentarisation des éleveurs peuls burkinabé et maliens, déjà présents dans le pays. 
4 Il ne s'agit pourtant pas de dresser le bilan de cette politique ambitieuse, mais de rappeler les efforts fournis entre 1974 et 1994 par la Côte d'Ivoire en vue de résoudre les problèmes de ravitaillement en viande du fait de la croissance continuelle des populations urbaines. En effet, deux tiers du bétail importé sont dirigés encore aujourd'hui sur Abidjan et Bouaké, les deux plus grands centres urbains ivoiriens (Brandt, 1995). L'année 1974 marque un tournant dans la politique d'importation et de commercialisation de viande dans ce pays qui a subi les contrecoups de la grande sécheresse. Cette sécheresse survenue au sein des pays fournisseurs de viande à la Côte d'Ivoire avait en effet occasionné une augmentation de $34 \%$ du prix du bétail à Abidjan. En prévision d'éventuels aléas du marché extérieur, les autorités ivoiriennes prendront une série de mesures. Tout d'abord, l'importation de viande qui était assurée par des sociétés commerciales étatiques fut exonérée de frais de douane entre 1975 et 1984. L'État ivoirien tenta ensuite au cours de cette même période de sédentariser les pasteurs peuls et de les engager davantage dans l'économie de marché ${ }^{2}$. Des infrastructures pastorales (barrages, bains détiqueurs, etc.) et des soins vétérinaires gratuits furent offerts aux Peuls dans le cadre de la politique d'accueil. La création de la Société pour le développement des productions animales (SODEPRA) inaugura cette politique réalisée avec l'aide internationale et qui visait l'autosuffisance de la Côte d'Ivoire en protéines animales.

Aménagement des zones d'accueil

5 Le ranch est un élevage intensif pratiqué dans un espace clos. A la différence du Nigeria, par exemple, où ce système avait été préconisé dans le projet de réforme de l'économie pastorale (Stenning 1959: 237), la Côte d'Ivoire opta au départ pour l'établissement de la SODEPRA ${ }^{3}$. Cet organisme, dont le fonctionnement correspondait au modèle institutionnel des Sociétés de développement (SODE), était chargé à l'instar de celles-ci de travailler à l'enracinement dans le pays d'une bourgeoisie locale devant prendre le relais pour poursuivre la production animale (Bernardet 1986:5). L'un des objectifs de la SODEPRA, créée en 1970 et subdivisée en deux zones, la SODEPRA-Centre (Bouaké) et la SODEPRA-Nord (Korhogo), était d'assurer aussi le développement de l'élevage traditionnel pratiqué dans le cadre villageois. Ainsi, le projet «taurin villageois ", lancé en direction des paysans propriétaires de bétail, sera complété en 1976 par «l'opération zébu» visant à fixer les éleveurs peuls. Cette opération, abandonnée à cause des déplacements des Peuls, tentait de contrôler ces éleveurs par le biais d'actions administratives (recensement), sanitaires (vaccinations) et commerciales (vente de médicaments).

6 L'aménagement des zones sylvo-pastorale de la Palé (200000 ha), située près de Boundiali, et agro-pastorale de Lokpoho (110000 ha), au nord de Korhogo, entrent aussi dans le cadre de la politique de sédentarisation des éleveurs peuls. Plus de deux tiers de la superficie de la zone sylvo-pastorale de la Palé aménagée en 1976, soit environ 130000 ha, étaient concernés par les aménagements pastoraux comportant, entre autres réalisations, des barrages, des bains détiqueurs, des puits, des magasins, des maisons, un laboratoire, etc. La Palé, qui devait accueillir plus de 30000 zébus, fut abandonnée par les éleveurs à cause de la réinfestation par la mouche tsé-tsé. Quant à la zone de Lokpoho, jugée favorable à l'élevage du zébu dès sa création, la SODEPRA-Nord avait prévu d'y développer des cultures fourragères. Mais cette approche mettant l'accent sur l'intégration de l'agriculture et de l'élevage se heurta au problème d'entretien des cultures fourragères, auquel s'ajouta la crainte des éleveurs 
devant les feux de brousse ${ }^{4}$. L'installation dans la zone de Lokpoho d'éleveurs n'a certes pas produit tous les résultats escomptés, mais elle offrit certains avantages aux paysans, notamment l'acquisition des bœufs de culture et de la fumure. Les leçons de l'échec de ces projets d'installation des pasteurs, réalisés à grands frais, ont été tirées. Certains sociologues expliquent l'échec de ces programmes par le désir des Peuls, ayant abandonné ces zones marginales, de se rapprocher des sociétés villageoises pour pouvoir commercialiser le lait (Bernardet 1984 ; Arditi 1990).

7 Il reste que la SODEPRA poursuivra ses actions de vulgarisation sur les différents aspects de la production animale, depuis la maîtrise jusqu'à la reproduction, en passant par les domaines de l'alimentation, de la santé et de l'exploitation des animaux. Entre 1982 et 1992, la direction régionale de la SODEPRA-Nord construisit des barrages et des lieux de vaccination, délimita des pistes à bétail et tenta de distribuer des semences de cultures fourragères à des prix subventionnés. A ces réalisations faites dans le cadre du Projet Aménagements Pastoraux (PAP) dans les départements qui composent la région Nord s'ajoutèrent des actions comme l'aménagement des micro zones et les tandems peul-sénoufo. Par la mise en place des micro zones, il s'agissait d'organiser l'espace de sorte qu'« un périmètre de quelques centaines d'hectares, vide de cultures, entoure le campement peuhl et assure une protection des cultures des villageois " ${ }^{5}$. La création de ces zones tampons, en favorisant l'intégration de l'agriculture et de l'élevage, devait contribuer à diminuer les conflits entre les Sénoufo et les Peuls. Les essais tentés cette fois encore n'ont guère été concluants. «La politique des tandem » consistait quant à elle à faire coopérer un éleveur peul et un agriculteur sur la terre appartenant à ce dernier, laquelle était divisée en deux parties. L'éleveur était chargé de fumer la première parcelle exploitée séparément avec son partenaire, cependant que la seconde était utilisée pour les ressources fourragères. Il s'agit en fait d'un système de rotation des cultures vivrières et fourragères, pour lequel les agriculteurs et les éleveurs manifestèrent de l'engouement, mais qui s'arrêta à la suite des affrontements de 1986 entre les Peuls et les Sénoufo de Dikodougou (sous-préfecture de Korhogo).

Des difficultés dues à une certaine indifférence des groupes, souvent peu motivés, amèneront par la suite les responsables du projet à infléchir leur approche. Cet infléchissement, qui s'est traduit par la mise en place d'Unités agro-pastorales (UAP) ${ }^{6}$, correspondait à la phase ultime de l'action de la SODEPRA qui, tout en s'attachant à préserver les infrastructures pastorales existantes, travailla à l'organisation des éleveurs. Cette phase préparatoire de désengagement des services publics du secteur de l'élevage est aussi celle durant laquelle le «Service transhumance UAP " s'efforça de collecter les informations sur le nombre de campements peuls, les déplacements saisonniers des troupeaux, la qualité des fourrages naturels (pâturages) et enfin l'élaboration d'un calendrier agro-pastoral. La SODEPRA, dissoute en 1994 à la suite de la restructuration du secteur agricole, fait maintenant partie de l'Agence nationale d'appui au développement rural (ANADER) ${ }^{7}$.

9 Avec la dissolution de la SODEPRA se tourne une page de l'histoire du pastoralisme en zone semi-humide ivoirienne. L'action et le mode d'intervention -de cette société sont controversés, et sa dissolution est même interprétée comme le signe d'échec de sa politique. Il ne s'agit pas de participer à la controverse, mais de remarquer que les efforts déployés durant deux décennies ont donné à la région Nord de la Côte d'Ivoire une vocation pastorale. Avec $65 \%$ du cheptel national, cette région est en effet la première en importance dans l'élevage bovin ${ }^{8}$. La densité du cheptel qui tourne autour 
de 19 bovins au km est comparable à celles que l'on observe au Mali et au Burkina. Dans cette région, 222 barrages, 65 puits, 33 bains détiqueurs et 11 marchés à bétail ont été construits. Selon les statistiques officielles, le cheptel bovin comptait en 1993 1,2 million de têtes, tous propriétaires confondus (paysans sénoufo, lobi, Peuls, etc.) ${ }^{9}$. L'évolution récente des relations entre les Sénoufo et les Peuls du département de Boundiali

Satisfaire les besoins de consommation de viande des populations urbaines reste l'objectif prioritaire des politiques et des développeurs. C'est ce qui explique l'abondance de la documentation sur les problèmes concrets de gestion du pastoralisme en Côte d'Ivoire. En revanche, la littérature sociologique sur les relations entre les éleveurs peuls et les agriculteurs sénoufo dans le contexte de la zone semi-humide ivoirienne est maigre comparativement à celle disponible sur certains Etats côtiers (Bénin, Nigeria ; etc.) où la présence peule est ancienne.

11 Les Sénoufo, dans le voisinage desquels vivent les Peuls, sédentaires ou transhumants, constituent le fond de peuplement le plus ancien de la région (Glaze 1981; Launay 1982). L'aire d'habitat des Sénoufo de la Côte d'Ivoire, qui couvre toute la région Nord, comprend une partie des régions Nord-Ouest (département d'Odienné) et Nord-Est (département de Bondoukou) de ce pays.

12 Bien qu'on y retrouve plusieurs groupes socio-fonctionnels (Kientz 1993), la société sénoufo reste dominée par les agriculteurs. Ceux-ci pratiquent à la fois les cultures vivrières (mil, maïs, riz, igname) et commerciales (arachide, coton). L'extension des cultures commerciales, et notamment celle du coton, est à la base de l'essor de la culture attelée. Dans les années 1960, la culture du coton, déjà ancienne dans le nord ivoirien, connut en effet un regain d'intérêt grâce à l'action de la Compagnie ivoirienne de développement des textiles (C.I.D.T.). Actuellement, plus de la moitié (soit 57\%) de la superficie exploitée du département de Boundiali utilise la traction bovine (Kientz 1993). L'utilisation de cette forme de technologie intermédiaire nécessite non seulement l'utilisation de produits fertilisants, mais aussi le recours aux Peuls, principaux fournisseurs de bœufs de culture aux Sénoufo.

13 L'élevage a peut-être été pratiqué de longue date par certains groupes sénoufo qui "ensevelissaient leurs morts dans des peaux de bœuf" (Arditi 1990: 143), mais aujourd'hui les Sénoufo ne sont pas à rigoureusement parler des éleveurs. Les paysans sénoufo que l'on qualifie rapidement d'éleveurs sont en réalité des propriétaires de bovins. Faute d'admettre que le fait de posséder des bœufs ne suffit pas à les qualifier pour l'activité d'élevage, les développeurs se sont évertués à comprendre l'attitude des Sénoufo, jugée bizarre, à l'égard du bétail. Chez ces derniers, les hommes ne s'occupent jamais du secteur de l'élevage. Les tentatives de valorisation des cultures fourragères en milieu paysan, qui n'ont pas produit les résultats escomptés, sont à rechercher de ce côté.

14 Selon la SODEPRA, l'effectif du cheptel bovin appartenant aux paysans sénoufo s'élevait en 1992 à environ 60507 têtes. Cet effectif représentant un peu plus de la moitié de la population bovine du département (119229 bovins) témoigne de l'importance du cheptel autochtone. Cette évaluation cache cependant des disparités sociales et régionales, car l'importance des bovins diminue du nord au sud. A ce facteur lié aux contraintes écologiques (mouche tsé-tsé) s'ajoutent des préoccupations (vols, dégâts de récoltes, etc.) qui conduisent certains paysans à renoncer à posséder des bœufs. 
15 Les bœufs jouent un rôle important dans la vie rituelle des Sénoufo, et notamment lors des funérailles. Les funérailles chez les Sénoufo font partie de ces rares occasions lors desquelles est consommée la viande du bœuf sacrificiel. Mise à part cette circonstance sociale et en dehors des activités de bouchers sénoufo isolés, la viande ramenée de la chasse, le porc, le mouton, le poisson, etc., restent les principales sources de protéines animales. La sous-consommation du lait reste également un phénomène général sauf chez certaines populations de la zone rizicole du département, qui accordent leur préférence à cet aliment consommé avec de la bouillie de riz. Ainsi donc, les efforts des projets qui visent l'augmentation de la productivité de viande et de lait restent encore des préoccupations urbaines extérieures au monde sénoufo d'autant plus que les paysans ont des sources de protéines animales autres que ces deux produits de l'élevage bovin.

16 Par ailleurs, l'importance accordée aux bovins dans la société sénoufo contemporaine tient à des motifs proprement économiques qui poussent de plus en plus de paysans à faire un placement en bœufs. Les paysans, qui négligent les produits de l'élevage bovin, s'accordent en effet pour souligner les avantages économiques de la possession des bœufs et les revenus qu'ils tirent occasionnellement de ce type d'investissement. D'après un avis répandu, les bœufs jouent un rôle d'épargne. Ainsi, en cas de mauvaises récoltes, le déficit agricole est-il compensé par le gain tiré de la vente de quelques têtes de bœufs.

17 Le cheptel villageois est généralement regroupé dans des parcs communautaires dont la gestion incombe aux Peuls. Un parc communautaire est formé par l'association de plusieurs petits propriétaires. Le premier paysan à avoir construit un enclos pour mettre ses animaux est le chef de parc à qui d'autres paysans du village, ou d'ailleurs, viendront remettre leurs bœufs. Le chef de parc, dont le rôle se limite aux tâches de coordination, reste en relation avec le berger après l'avoir recruté. La responsabilité du parc ne confère cependant aucun privilège à celui qui en est le chef.

18 Dans certains villages, la moitié du cheptel bovin sénoufo est gardée dans des parcs communautaires dont la moyenne est de quatre propriétaires par parc. Ces proportions reflètent la situation générale des parcs communautaires dans l'ensemble du nord ivoirien.

19 L'obligation de recourir aux Peuls, doués de compétence dans la gestion des bovins, fonde la division ethnique du travail entre ceux-ci et les Sénoufo. L'élevage est reconnu être la spécialité des Peuls (S. flabele). Le salaire, la consommation et la commercialisation du lait sont les principaux avantages que les bouviers peuls tirent de leur service chez les Sénoufo. En définitive, la division du travail entre les bergers peuls et les Sénoufo épargne les paysans de deux soucis majeurs : le vol des bœufs et les dégâts qu'ils commettent dans les champs. Le second problème, évoqué plus loin, constitue le motif principal de démêlés entre les Sénoufo et les éleveurs peuls propriétaires d'importants troupeaux.

20 - Les Peuls: le département de Boundiali est le lieu de résidence de plusieurs familles d'éleveurs. Les peuls burkinabé y sont plus nombreux que leurs homologues maliens. L'installation dans le nord de la Côte d'Ivoire ${ }^{10}$ des éleveurs peuls burkinabé, dont il est ici question, remonte au début des années 1960. Leurs déplacements en zone semi-humide ivoirienne s'inscrit dans deux courants migratoires opposés dans leur orientation, mais convergents dans leur finalité. C'est au cours des années 1950 que commença la migration pastorale de ces Peuls partis de l'ancienne chefferie de Barani 
(nord-ouest du Burkina Faso). Les premiers groupes, dirigés vers le Mali, s'installèrent d'abord dans la région de San. Ils amorcèrent ensuite une descente dans les provinces méridionales de Koutiala et de Sikasso pour finalement pénétrer sur le territoire de la Côte d'Ivoire où ils fondèrent, en 1964, le village de Foulabougou («village des Peuls ») dans le département frontalier de Tengréla. Ce mouvement fut poursuivi jusqu'aux approches de Boundiali. A ce courant migratoire, initial et indirect, succédera une migration intentionnelle. Celle-ci concerne principalement les éleveurs entrés .dans le nord de la Côte d'Ivoire par le sud du Burkina (région de Niangologo).

Il n'existe pas de contrat d'installation entre les éleveurs peuls et les Sénoufo. Pour obtenir le droit d'installation, les Peuls doivent discuter avec les chefs de village sénoufo (S. fanfolo) ou avec les responsables de la terre (S. tarfolo) ${ }^{11}$. Les leaders peuls ne paient rien aux sénoufo, mais il arrive que certains d'entre eux offrent aux chefs sénoufo des cadeaux en signe de reconnaissance. Les éleveurs peuls vivent dans des campements (F. gure) dispersés dans le département de Boundiali. Le campement, composé de plusieurs familles, est sous le contrôle de l'homme le plus âgé (F. mawdo).

Outre l'élevage bovin, qui reste leur activité économique principale, les Peuls sédentarisés pratiquent l'agriculture (maïs, sorgho) dans le cadre de la subsistance familiale. Ils possèdent à la fois des zébus, des taurins ( $\mathrm{N}^{\prime}$ dama) et du bétail trypanotolérant. Les zébus, appelés seenooji en raison de leur origine sahélienne ou Seeno (nord du Burkina), résistent aux longues marches. Mais, à la différence des N'dama, ils sont sensibles à l'humidité et à la trypanosomiase (F. powè), principale contrainte pesant sur l'élevage dans le nord ivoirien. L'élevage bovin en zone semi-humide nécessite non seulement le recours aux médicaments, mais aussi les croisements (zébu X N'dama), méthode de contrôle de la trypanosomiase pratiquée par les éleveurs peuls. Le produit du premier croisement, appelé worsooji, est utilisé pour effectuer un croisement de deuxième génération (worsooji $\mathrm{X}$ zébu). La sous-espèce bovine de la deuxième génération a, selon les éleveurs, une haute productivité en lait outre le fait qu'elle conserve beaucoup de ressemblance avec le zébu, élément constitutif de l'identité peule dans la condition humide.

Le bétail constitue la richesse (F. jawdi) des éleveurs peuls. Le nombre du cheptel bovin détenu par les plus riches d'entre eux s'élève parfois à 2000 têtes de bœufs. La gestion d'un gros cheptel nécessite sa division en troupeau laitier et troupeaux de brousse. Le troupeau laitier, habituellement gardé à proximité du campement, produit le lait pour la consommation familiale et dont une partie est commercialisée. La surveillance des troupeaux de brousse repose sur l'exploitation d'un salariat formé par des bergers peuls, jeune et célibataires pour la plupart compte tenu de la dureté de leurs conditions de vie et travail.

Dégâts de cultures et problèmes fonciers

24 Les éleveurs peuls et les paysans sénoufo entretiennent des relations à la fois conflictuelles et complémentaires. Ainsi, les Peuls sont encore les principaux fournisseurs de bœufs de culture aux Sénoufo. Le zébu ainsi que la sous-espèce bovine issue du croisement tirent bien la charrue. Ne voulant pas se séparer des zébus qu'ils considèrent comme un élément de leur identité, les Peuls vendent plutôt les bovins croisés aux paysans. Nombreux sont également les Sénoufo qui, lors des funérailles, achètent des bœufs dans les campements peuls avoisinants.

25 Ces relations complémentaires sont cependant de plus en plus contrebalancées par la situation de tension actuelle. La garde des bœufs des paysans par les éleveurs peuls ou 
le contrat de fumure, pour ne citer que ces deux formes classiques de complémentarité et de coopération entre pasteurs et paysans, sont en déclin dans certaines zones du département de Boundiali. La situation dans certaines localités est si tendue que les Peuls cherchent à ne pas créer de difficultés supplémentaires. Des éleveurs peuls refusent, par exemple, de garder les bœufs des paysans pour éviter d'être accusés plus tard de détournement. A cette situation s'ajoutent les démêlés continuels au sujet des dégâts de cultures et de récoltes, principal facteur de tensions entre les éleveurs peuls et les agriculteurs sénoufo.

La non-protection des cultures, le stockage des récoltes dans les champs ainsi que l'augmentation du cheptel bovin des Peuls expliquent la facilité avec laquelle interviennent ces dégâts. Le manque de moyens matériels et financiers des administrations territoriales (sous-préfectures) entraînant une lenteur dans les constats et les dédommagements des dégâts contribuent aussi à augmenter la frustration des paysans. Les doutes et les critiques exprimées à l'égard des autorités sous-préfectorales, dont la compétence et l'honnêteté sont en cause, pose un problème de confiance. C'est pourquoi les disputes entre les paysans et les éleveurs sont de plus en plus réglés au niveau local avec l'aide des chasseurs (S. donzobele). C'est l'incapacité de l'Etat ivoirien d'assurer la sécurité dans la région Nord marquée par la recrudescence du grand banditisme qui a d'abord poussé les préfets à solliciter la collaboration des chasseurs. Armés de fusils de chasse traditionnels, ils font pendant la nuit des tournées de police dans les villages soumis, pour ainsi dire, au couvre-feu. Sollicités ensuite par les paysans, leur rôle consiste à rechercher les bergers ayant commis des dégâts dans les champs situés à l'intérieur des limites du territoire placé sous leur contrôle. Les chasseurs sénoufo sont aussi chargés de notifier aux éleveurs l'interdiction de passer et de faire paitre les bœufs près du village. Les punitions infligées aux bergers peuls et qui sont souvent disproportionnées aux dégâts commis ont révélé les abus de pouvoir des équipes de chasseurs qui ont tendance à se substituer à l'administration. En dépit des accusations dont elle fait l'objet, l'administration reste encore un intermédiaire privilégié dont la médiation, sollicitée dans bien des cas, est indispensable.

27 Cependant, les problèmes de dégâts de cultures ne sont pas le seul facteur explicatif des conflits entre les éleveurs et les paysans ${ }^{12}$. Il existe dans les zones de forte pression démographique une situation de tension, résultat de la concurrence entre les éleveurs et les agriculteurs pour les ressources (eau, terre, etc.). Cette concurrence pousse les autochtones de certaines zones à déplacer ou à expulser de leur terroir les éleveurs en vue de s'assurer le contrôle des ressources en entier. Ce phénomène s'observe surtout dans les villages peuplés de la partie septentrionale du département de Boundiali où les densités rurales $(20-45$ hab. $/ \mathrm{km})$ sont les plus élevées ${ }^{13}$. Ainsi, les paysans ont-ils pratiquement réussi à expulser de leur village les éleveurs.

Dans d'autres localités, les tentatives paysannes se sont soldées par des agressions contre les Peuls en 1993. Ailleurs enfin, la marginalisation des éleveurs se traduit par l'interdiction expresse d'accéder aux barrages. L'accès aux barrages, SODEPRA ou privés, est en principe gratuit dans le nord ivoirien. Les paysans qui s'opposent maintenant à leur utilisation considèrent ces points d'eau comme un droit exclusif de leur village. D'autres voient les barrages comme des réalisations pour des étrangers peuls. C'est sans doute pour cela aussi que certains d'entre eux ne se font pas faute de cultiver à proximité des barrages. Ce faisant, ils violent le principe qui veut qu'une 
distance d'un kilomètre sépare les champs du barrage. A Boundiali même l'impossibilité d'accéder au barrage tient surtout aux activités des femmes travaillant dans le cadre d'une coopérative maraîchère. Les actions des comités de surveillance des barrages, composés de paysans choisis par le chef de village, provoquent parfois des conflits entre habitants d'une même localité. C'est surtout en saison sèche que le problème d'eau se pose avec acuité pour les hommes et les animaux. Des éleveurs se sont vus interdire l'accès aux barrages sous prétexte que les bœufs font fuir les poissons qui s'y trouvent.

L'enclavement de plusieurs campements peuls est un autre problème crucial que pose l'extension des cultures. Des paysans, à la recherche de terres, n'hésitent plus en effet à venir cultiver autour des campements. Cette situation oblige les Peuls à éloigner leur troupeau laitier de ces résidences enclavées pour éviter les problèmes de dégâts.

Il existe aussi une concurrence entre agriculteurs. De nos entretiens, il résulte que la pénurie d'eau, la disponibilité des terres cultivables et la disparition progressive des arbres sont les trois problèmes débattus en priorité par les hommes et les femmes de plusieurs villages du département de Boundiali ${ }^{14}$. Or la présence d'un cheptel important contribue à compliquer davantage la situation. Le problème soulevé par les paysans est moins le manque d'espace ${ }^{15}$ que celui de la disponibilité des terres fertiles. Avec l'extension des cultures commerciales et la pression démographique on note un recours croissant aux produits fertilisants (Pradeep 1992). Mais cette solution tend à être limitée dans certaines localités faute de moyens financiers.

Les stratégies paysannes, individuelles ou collectives, visant à s'approprier l'espace et les ressources, poussent les éleveurs à solliciter continuellement la médiation de l'administration. Les rivalités entre les deux groupes ont déjà provoqué le repli de certains peuls sur le Mali, mais aussi l'émergence d'un courant migratoire vers le Ghana, dont nous ignorons pour le moment les phases et les modalités. Des propriétaires peuls ayant pris la mesure de l'enjeu procèdent aussi à une répartition de leur cheptel dont une partie est envoyée au Mali durant la saison pluvieuse. Ces troupeaux ne regagnent la Côte d'Ivoire qu'en début de saison sèche. L'évolution récente des relations entre les Peuls et les Sénoufo a bien entendu attiré l'attention de l'État ${ }^{16}$. Mais le cadre limité de cette réflexion ne permet pas d'examiner en détailles solutions proposées en vue de réduire les conflits, les tensions et les incompatibilités. Nous nous limiterons donc au rôle du foncier en tant que facteur de médiation entre les Sénoufo et les Peuls.

Le foncier comme facteur de médiation.

En Côte d'Ivoire, comme ailleurs, le problème foncier est la contrainte majeure de bon nombre de projets de développement, et plus particulièrement dans les programmes d'installation des groupes. A l'origine de ce problème se trouve surtout l'antagonisme entre le droit foncier moderne et le droit foncier traditionnel. Le pouvoir foncier de l'Etat qui prétend être le propriétaire éminent de l'espace national est en réalité nominal et théorique. Pour la région que nous considérons, la terre est gérée encore aujourd'hui de façon coutumière. Le système foncier sénoufo, rigide dans son principe, diffère en effet de ceux que l'on observe ailleurs dans d'autres régions ivoiriennes ${ }^{17}$. L'idéologie et la pratique foncières des Sénoufo sont incompatibles avec toute spéculation sur la terre qui ne peut être ni vendue ni échangée. La forme d'appropriation de la terre en vigueur est de type communautaire. Chaque patrilignage et matrilignage propriétaire d'une portion de la terre, au sens traditionnel, exerce un 
droit d'usage inaliénable. Son accès est accordé par le chef de lignage à tout individu appartenant à la communauté. Pour ce qui concerne les étrangers, demandeurs de terrains de culture, on ne leur refuse jamais l'accès à la terre. Seulement, son utilisation se limite au privilège usufruitier. L'interdiction de planter un arbre ou de creuser un puits fait d'ailleurs partie du mécanisme social de contrôle visant à empêcher toute velléité de revendication par les nouveaux venus d'un droit de propriété de la terre. $C^{\prime}$ est avec les permissions consenties par les Sénoufo aux éleveurs peuls en vertu de ces principes que ces derniers se sont donc installés sur les lisières des villages d'accueil. Mais la poussée démographique actuelle pousse les autochtones, ayant autrefois accordé l'hospitalité aux éleveurs, à vouloir récupérer leurs terres. Ces problèmes de terres ont déjà provoqué de nombreux conflits fonciers entre les Sénoufo du département de Boundiali ${ }^{18}$.

La saturation foncière que connaissent certaines zones du département de Boundiali renvoie pourtant à un problème plus général car cette crise est propre à l'ensemble du nord ivoirien. C'est pour trouver une solution à ces problèmes fonciers que le gouvernement vient de lancer le projet du Plan foncier rural (P.F.R.). Ce projet, qui s'inscrit dans le cadre du Programme national de gestion de l'espace rural (PNAGER) ${ }^{19}$ est chargé de recenser les terres avec l'aide des populations paysannes. En cherchant à lutter contre le "gaspillage foncier", les principaux bailleurs de fonds du projet (Banque mondiale, CFD, FAC, etc.), veulent créer un marché foncier après avoir garanti la sécurité foncière des paysans.

Outre cette fonction visant à accumuler les richesses, il semble que le P.F.R. pourra remplir une autre fonction: 'celle de pacificateur de la situation de tension entre les Peuls et les Sénoufo. Selon les promoteurs du projet, le P.F.R. donnera en effet un caractère contractuel aux relations entre les deux groupes qui parviendront à une entente. Enfin, un autre aspect de l'intervention du P.F.R. concerne les indications sur le choix de l'emplacement des barrages devant être construits ultérieurement par l'ANADER. Il s'agit d'éviter de créer de nouveau des situations conflictuelles, car bon nombre des barrages actuels ont semble-t-il été construits sans que les chefs de village aient été pleinement associés.

Compte tenu de la diversité de cas, les relations entre les Peuls et les Sénoufo de Boundiali ne sauraient être généralisées à l'ensemble de la région Nord. Cependant, la situation dans ce département donne une impression d'antagonisme entre deux groupes: d'un côté, l'Etat et les couches urbaines ${ }^{20}$ (fonctionnaires, députés, développeurs, etc.) intéressés pour diverses raisons aux bénéfices de la présence peule et, de l'autre, une majorité de paysans de plus en plus embarrassés par cette présence ${ }^{21}$. La pression démographique et les dégâts de cultures que provoque la présence d'un cheptel important sont les facteurs explicatifs de l'embarras actuel des sénoufo hostiles à la présence peule sur des terroirs aux ressources surexploitées. Cette hostilité des paysans, dont on a de fortes raisons de penser qu'elle restera dans les années à venir un défi pour les autorités politiques, empêcherait le renforcement de la vocation pastorale de la Côte d'Ivoire si aucune solution satisfaisante n'est apportée à la question de l'intégration des Peuls et à la crise foncière touchant la région Nord. 


\section{BIBLIOGRAPHIE}

Abruzzi, William S. 1982 « Ecological Theory and Ethnic Differentiation among Human Populations », Current Anthropology, vol 23 (1) : 13-35.

Arditi, Claude 1990 «Les Peul, les Senufo et les vétérinaires : pathologie d'une opération de développement dans le nord de la Côte d'Ivoire ", Cahiers des Sciences Humaines, 26 (1-2) : 137-153.

Barry, Mody B., Ouattara, Niho, Touré, Moussa \& Marty, André 1994 Bilan des actions pastorales et appui à l'élaboration d'un programme à moyen terme en République de Côte d'Ivoire (aide mémoire provisoire), Ministère de l'Agriculture et des Ressources Animales, Fonds d'Aide et de Coopération, $7 \mathrm{p}$.

Barth, Fredrik., ed. 1969 Ethnic Groups and Boundaries. The Social Organisation of Culture Difference, London, Bergen.

Barth, Fredrik, 1994 « Enduring and Emerging Issues in the Analysis of Ethnicity ", in H. Vermeulen \& C. Govers, eds, The Anthropology of Ethnicity. Beyond "Ethnic Groups and Boundaries", Amsterdam, Het Spinhuis : 10-32.

Basler, Alois, 1987 « Agrar-und Entwicklungspolitik im Konflikt. Eine Fallstudie der Rindfleischversorgung der Côte d'Ivoire », Agrarwirtschaft, 37 (7-8) : 220-228.

Basset, Thomas, 1994 « Hired Herders and Herd Management in Fulani Pastoralism (Northem Côte d'Ivoire) », Cahiers d'Etudes africaines, 133-135, XXXIV (1-3) : 147-173.

Bernardet, Philippe, 1984 Association agriculture-élevage en Afrique. Les Peuls semi-transhumants de Côte d'Ivoire, Paris, L'Harmattan (Collection « Alternatives paysannes »).

Bernardet, Philippe, 1986 « Eleveurs, agriculteurs et assistance technique en Côte d'Ivoire. Les épreuves d'une rencontre ", Communication présentée au $13^{e}$ congrès européen de sociologie rurale, Braga.

Bierschenk, Thomas, 1993 « The Creation of a Tradition. Fulani Chiefs in Dahomey/Bénin from the Late 19th Century ", Paideuma, $39: 1-22$.

Brandt, Harmut, 1995 Auswirkungen von Exporterstattungen der Europaischen Union auf die Rindjleischsektoren westafrikanischer Liinder, Berlin, Deutsches Institut flir Entwicklungspolitk.

Defaye, Jean Bernard \& Coulibaly, Zié. 1992 Rapport annuel 1992, Projet U.A.P., Service intégration de la transhumance, SODEPRA-Nord, Korhogo.

Diallo, Youssouf, 1995 « Paysans, pasteurs et intervention étatique : analyse préliminaire des problèmes et de l'évolution du pastoralisme dans le département de Boundiali (Côte d'Ivoire) »University of Bielefeld, Working Paper n²25 (Africa programme).

Dozon, Jean-Pierre, 1985 « Les Bété : une création coloniale », in J.-L. Amselle \& E. M'Bokolo, eds, Au cœur de l'ethnie. Ethnies, tribalisme et Etat en Afrique, La découverte : 49-85.

Ezeomah, Chimah, 1987 « The Settlement Problems of Nomadic Fulani in Nigeria », Rural Africana, 27 : 35-45.

Forster, Till, 1987 « Der Poro-Bund der Senufo heute. Institutionen segmentärer gesllschaften und entwicklungspolitische Massnahmen », Baessler-Archiv, Neue Folge, Band XXXV : 191-220. 
Frantz, Charles, 1978 " Ecology and Social Organization Nigerian Fulbe (Fulani)", in W. Weissleder, ed, The Nomadic Alternative. Modes and Models of Interaction in the African-Asian deserts and Steppes, The hague, Paris, Moutoun Publishers : 97-118.

Galaty, John G., 1981 « Introduction : Nomadic Pastoralists and Social Change- Processes and Perspectives ", in J. G. Galaty \& P. C. Salzman, eds, Change and Development in Nomadic and Pastoral Societies, Leiden, E. J. Brill : 4-26.

Glaze, Anita J., 1981 Art and Death in a Senufo Village, Bloomington, Indiana University Press.

Hopen, C. Edward, 1958 The Pastoral Fulbe Family in Gwandu, London, Ibadan, Accra, Oxford University Press.

Hyden, Goran, 1986 « The Anomaly of the African Peasantry ", Development and Change, vol. 17 : 677-705.

Kientz, Albert, 1993 Développement rural du Département de Boundiali, Côte d'Ivoire. Bilan-diagnostic, G.T.Z.

Lachenmann, Gudrun, 1986 « Rural Development in Mali- Destabilisation and Social organisation », Quarterly Journal of International Agriculture, Vo125 (3) : 217-233.

Launay, Robert, 1982 Traders without trade. Responses to change in two Dyula communities, Cambridge, Cambridge University Press.

Mafeje, Archie, 1985 « Peasants in Sub-Saharan Africa », Afrique et développement, vol X (3) : 28-37.

Ministère de l'Agriculture et des Ressources Animales, 1994 Atelier sur les conflits agriculteurs-éleveurs (7,8,9 juillet 1994), Yamoussoukro (document préparatoire, version provisoire du 16.06.94).

Oxby, Clare, 1984 « Settlement Schemes for Herders in the Subhumid Tropics of West Africa : Issues of Land Rights and Ethnicity ", Development Policy Review, vol 2 : 217-233.

Post, Ulli, 1993 « Nur Schaden, kein Nutzen : Die EG-Rindfleischexporte nach Westafrika », Entwicklung und ländlicher Raum, 27 (6) : 2.

Pradeep, Itty, 1992 Economics of Village Cattle Production in Tsetse Affected Areas of Africa. A Study of Trypanosomiasis Control Using Cattle and Chemotherapy in Ethiopia, Kenya, Côte d'Ivoire, The Gambia, Zaïre and Togo, Hartung-Gorre Verlag, Konstanz.

Roos, Günter, 1995 L'élaboration des premiers fondements d'une stratégie de promotion de la gestion communautaire des ressources naturelles dans le département de Boundiali, Côte d'Ivoire, Projet D.R.D.B.-GTZ, Giessen.

Schlee, Günther, 1994 Identities on the Move. Clanship and pastoralism in Northern Kenya, Nairobi, Gideon S. Were Press (1re ed. 1989).

Stenning, Derrick J., 1959 Savannah Nomads. A study of the Wodaabe Pastoral Fulani of Western Bornu Province Northern Region, Nigeria, London, Ibadan, Accra, Oxford University Press.

Tonah, Steve, 1993 The Development of Agropastoral Households in Northern Ghana : Policy Analysis, Project Appraisal and Future Perspectives, Saarbrücken, Fort Lauderdale ("Bielefeld Studies on the Sociology of Development").

Université d'Abidjan, Institut d'Ethno-Sociologie (LE.S.), 1975 L'élevage peul dans le nord de la Côte d'Ivoire (étude sociologique), Abidjan. 


\section{NOTES}

1.Les informations présentées ici ont été collectées lors d'une enquête de terrain de trois mois réalisée en 1994 dans le département de Boundiali (nord de la Côte d'Ivoire). Cette enquête s'inscrit dans le cadre des travaux du Centre de recherche en sociologie du développement (Université de Bielefeld) sur le thème "Etat, marché, ethnicité". 2.La création de marchés de bétail et la construction de stations d'engraissement et d'abattoirs frigorifiques dans le nord du pays répondent à cette préoccupation de contrôle. En 1976 a été installée à Ferkéssédougou une station d'engraissement à proximité de laquelle sera construite en 1980 un abattoir frigorifique. La station de Ferkéssédougou privatisée en 1991 et dont la capacité est d'environ 12000 têtes par an est exploitée par la Société d'exploitation du bovin et de la viande (SEBOVIA).

3.Le ranch de la Marahoué est en effet l'un des rares projets de ce type à être institué en Côte d'Ivoire.

4.Une situation comparable est décrite par Oxby (1984:225).

5.Ministère de l'agriculture et des ressources animales 1994, p. 6.

6.Le financement de cette phase du projet a surtout été assuré par le Fond d'aide et de coopération (FAC) et la Caisse française de développement (CFD).

7.Font également partie de cette institution privée la Compagnie ivoirienne de développement des cultures vivrières (C.I.D.V.) et la Société d'assistance technique pour la modernisation de J'agriculture en Côte d'Ivoire (SATMACI).

8.Les trois autres régions sont, par ordre d'importance, le Nord-Est (Bondoukou, Bouna) avec $14 \%$ du cheptel national, le Centre Nord (Katiola, Bouaké, Dabakala) avec 9\% et le Nord-Ouest (Mankono, Odienné, Séguéla) avec 7\% (Source : Ministère de l'agriculture et des ressources animales 1994). Chaque région est une entité administrative composée de plusieurs départements.

9.Dans la région Nord, la densité du cheptel est la suivante : Tengréla 29 bovinslkm, Korhogo 23, Boundiali 16 et Ferkéssédougou 13.

10.La région Nord, zone d'implantation principale des éleveurs peuls originaires du Mali et du Burkina, est composée des départements de Boundiali, Tengréla, Korhogo et Ferkéssédougou.

11.Le terme folo sert à désigner le chef, le responsable ou le possesseur de quelque chose ; lai : signifie la terre au sens large.

12.Pour l'ensemble du département de Boundiali, le Bureau des affaires domaniales rurales (B.A.D.R.) a enregistré en 1992-1993 192 ha de cultures détruites dont le coût a été estimé à 5784672 francs CFA.

13.Avec une moyenne de $16 \mathrm{hab}$. $/ \mathrm{km}$, la région de Boundiali figure pourtant parmi les départements les moins densément peuplés de Côte d'Ivoire.

14.Cf. aussi G. Roos (1995).

15.La caractéristique du peuplement dans le département de Boundiali est en effet l'inégale répartition de sa population : la zone occidentale dépeuplée, ayant un aspect montagneux, s'oppose à la zone orientale où se concentre une fraction importante de la population et qui renferme des noyaux de peuplement se situant entre 20 et 40 hab/ $\mathrm{km}$.

16.En juillet 1994 eut lieu à Yamoussoukro une conférence à ce sujet. Voir Atelier sur les conflis agriculteurs éleveurs (7, 8, 9 juillet 1994), Yamoussoukro (document préparatoire, version provisoire du 16.06.94). 
17.Par exemple, dans le Centre-Ouest ivoirien, notamment en pays bété, la terre est un bien aliénable. La facilité d'accès à la terre et sa transformation en marchandise, dans le cadre de l'économie de plantation, ont provoqué un renversement de situation entre les autochtones bété et les allochtones (Baule, Burkinabé, etc.). Voir sur ce point Dozon (1985).

18.A L'origine des violents conflits qui ont opposé en 1993 les habitants de Katanra (sous-préfecture de Gbon) à ceux de Kpafonon (sous-préfecture de Kolia) se trouvent les problèmes de terres de culture. La même année survenaient des conflits fonciers entre les villages de Boyo et Timboroni.

19.Le PNAGER cherche à relancer les investissements collectifs en milieu rural.

20.Un tiers des barrages, sur la quarantaine que compte le département de Boundiali, a été construit par des députés ou des fonctionnaires qui investissent de plus en plus dans le secteur de l'élevage bovin.

21. Ce type d'antagonisme se retrouve ailleurs dans d'autres pays côtiers, et notamment au Ghana. A la suite de problèmes avec les paysans en 1988, les autorités administratives ont forcé les éleveurs peuls à partir du pays (Tonah 1993). Cette décision administrative a provoqué une importation massive de viande de l'extérieur, et surtout de l'Europe, en vue de résoudre le problème de ravitaillement de viande des couches urbaines qu'une telle décision ne pouvait manquer d'aggraver.

\section{AUTEUR}

\section{YOUSSOUF DIALLO}

Sociology of Development Research Centre, Postf. D-33501 Bielefeld, Allemagne 